\title{
Intermittent fasting increases the expressions of SODs and catalase in granule and polymorphic cells and enhances neuroblast dendrite complexity and maturation in the adult gerbil dentate gyrus
}

JI HYEON AHN ${ }^{1 *}$, BICH NA SHIN ${ }^{2 *}$, MINAH SONG $^{3}$, HYUNJUNG KIM $^{3}$, JOON HA PARK $^{1}$, TAE-KYEONG LEE ${ }^{3}$, CHEOL WOO PARK ${ }^{3}$, YOUNG EUN PARK ${ }^{3}$, JAE-CHUL LEE $^{3}$, JUN-HWAN YONG ${ }^{4}$,

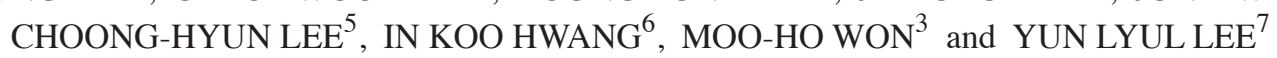

\begin{abstract}
${ }^{1}$ Department of Biomedical Science and Research Institute for Bioscience and Biotechnology, Hallym University, Chuncheon, Gangwon 24252; ${ }^{2}$ Danchunok Company, Chuncheon, Gangwon 24210; ${ }^{3}$ Department of Neurobiology, School of Medicine, Kangwon National University, Chuncheon, Gangwon 24341; ${ }^{4}$ Department of Occupational Therapy, Dongnam Health University, Suwon, Gyeonggi 16238; ${ }^{5}$ Department of Pharmacy, College of Pharmacy, Dankook University, Cheonan, Chungcheongnam 31116; ${ }^{6}$ Department of Anatomy and Cell Biology, College of Veterinary Medicine, and Research Institute for Veterinary Science, Seoul National University, Seoul 08826;

${ }^{7}$ Department of Physiology, College of Medicine and Institute of Neurodegeneration and Neuroregeneration, Hallym University, Chuncheon, Gangwon 24252, Republic of Korea
\end{abstract}

Received September 5, 2018; Accepted December 31, 2018

DOI: $10.3892 / \mathrm{mmr} .2019 .9822$

\begin{abstract}
Intermittent fasting ( $\mathrm{ImF})$ is known to reduce oxidative stress and affects adult neurogenesis in the hippocampal dentate gyrus. However, it is unknown how ImF affects endogenous antioxidants expressions, cell proliferation, and neuroblast differentiation and their dendrite remodeling over 3 months in the dentate gyrus of adult gerbils. The present study subjected 6-month old male gerbils to a normal diet or alternate-day ImF for 1, 2 and 3 months. Changes in body weight were not significantly different between gerbils fed a normal diet and on ImF. The present study also investigated the effects of ImF on antioxidant enzymes [superoxide dismutase (SOD)-1, SOD2 and catalase] using immunohistochemistry, and endogenous cell proliferation,
\end{abstract}

Correspondence to: Professor Moo-Ho Won, Department of Neurobiology, School of Medicine, Kangwon National University, 1 Gangwondaehak, Chuncheon, Gangwon 24341, Republic of Korea E-mail:mhwon@kangwon.ac.kr

Professor Yun Lyul Lee, Department of Physiology, College of Medicine and Institute of Neurodegeneration and Neuroregeneration, Hallym University, 1 Hallymdaehak, Chuncheon, Gangwon 24252, Republic of Korea

E-mail: yylee@hallym.ac.kr

*Contributed equally

Key words: intermittent fasting, superoxide dismutase, catalase, cell proliferation, neuroblast differentiation, dentate gyrus neuroblast differentiation and neuroblast dendrite complexity by using Ki67 (a cell proliferation marker) and doublecortin (neuroblast differentiation marker) immunohistochemistry in the dentate gyrus. SOD1, SOD2 and CAT immunoreactivities were shown in cells in the granule cell and polymorphic layers. SOD1, SOD2 and catalase immunoreactivity in the cells peaked at 2, 1 and 1 month, respectively, following ImF. Cell proliferation was $\sim 250,129$ and $186 \%$ of the control, at 1,2 and 3 months of ImF, respectively. Neuroblast differentiation was $\sim 41,32$ and $12 \%$ of the control, at 1, 2 and 3 months of ImF, respectively, indicating that dendrites of neuroblasts were more arborized and developed at 3 months of ImF. Taken together, these results indicate that $\mathrm{ImF}$ for 3 months improves endogenous SOD1, SOD2 and catalase expressions and enhances cell proliferation, and neuroblast dendrites complexity and maturation in the adult gerbil dentate gyrus.

\section{Introduction}

Intermittent fasting ( $\mathrm{ImF}$ ) or intermittent caloric restriction refers to the dietary restriction that reduces food intake during a defined period (1). Previous studies have revealed positive effects of dietary restriction. For example, ImF improves the tolerance of neurons against brain ischemic damage in mice and rats $(2,3)$. Furthermore, dietary restriction ameliorates age-related decline of presynaptic proteins (i.e., synaptophysin) in the rat hippocampus (4), and caloric restriction increases hippocampal-dependent spatial learning memory in mice (5), suggesting that ImF improves spatial learning memory.

Neurogenesis in adults is characterized by proliferation and differentiation of a small number of neural progenitor 
cells (NPCs) into mature neurons (granule cells) or glial cells (astrocytes or oligodendrocytes), which occurs in the subgranular zone (SgZ) of the hippocampal dentate gyrus $(6,7)$. The processes of the adult neurogenesis include proliferation, differentiation, morphogenesis and maturation of adult-born neurons, and integration of newborn neurons into the neural circuitry of the hippocampus (8). It has been reported that dietary restriction regimen can enhance cell proliferation and differentiation of NPCs through upregulation of neurotrophic factors, such as brain-derived neurotrophic factor and neurotrophin-3 in the hippocampus $(9,10)$.

Superoxide dismutases (SODs) such as $\mathrm{Cu} / \mathrm{Zn} \mathrm{SOD}$ (SOD1) and Mn SOD (SOD2) are one of major antioxidant enzymes involves in removing superoxide anion radicals (11), and catalase (CAT) is a common enzyme as a $\mathrm{H}_{2} \mathrm{O}_{2}$ scavenger (12). A previous study has reported that predominant expression of SOD1 is shown in early neural precursors and migrating neuroblasts in the $\mathrm{SgZ}$ (13). In addition, expression level of CAT mRNA is maintained during all three stages of neural differentiation (undifferentiated cells, embryoid bodies, and post-plating) in embryonic stem cells as an in vitro model for neural differentiation (14). In this regard, antioxidant enzymes play important roles in the process of neurogenesis.

Despite many researches on beneficial effects of $\mathrm{ImF}$, studies on cell proliferation and the differentiation of neuroblasts including their dendrite remodeling according to the period of ImF in the hippocampal dentate gyrus remains to be elucidated. Thus, in the present study, we investigated effects of ImF for 1 to 3 months on expressions of Ki67 (a cell proliferation marker) and doublecortin (DCX, a neuroblast differentiation marker), and the complexity of neuroblast dendrites in the dentate gyrus of the gerbil hippocampus. In addition, we examined changes in expressions of endogenous antioxidant enzymes such as SOD1, SOD2, and CAT in the dentate gyrus to study their related mechanisms of ImF in the cell proliferation and neuroblast differentiation.

\section{Materials and methods}

Experimental animals. Male gerbils were obtained at six months of age (body weight, $65 \pm 4.6 \mathrm{~g}$ ) from the Experimental Animal Center, Kangwon University, Chuncheon, Republic of Korea, and maintained at a constant temperature $\left(23 \pm 0.4^{\circ} \mathrm{C}\right)$ and humidity $(50 \pm 0.6 \%)$ with a 12 -h light/dark cycle. The process of handling and caring animals conformed to the guidelines being in compliance with current international laws and policies (NIH Guide for the Care and Use of Laboratory Animals, The National Academies Press, 8th edition, 2011). The protocol of this experiment was approved by the Institutional Animal Care and Use Committee (IACUC) at Kangwon National University (approval no. KW-180124-1; Gangwon, Republic of Korea). No animals died during this experiment.

Im $F$ and experimental groups. Animals were fed commercially available rodent normal diet or ImF (provided food on alternate days) was applied for 1, 2, and 3 months according to published methods $(1,2,15)$. Food intake of ImF group was controlled daily ( $10 \mathrm{~g}$ per day), and body weight of normal diet and ImF groups was monitored every month. Animals with normal diet or ImF were randomly assigned to following groups: i) Control group ( $n=7)$, which was allowed free access to water and food; ii) 1-month (1-M) $\operatorname{ImF}$ group (n=7); iii) 2-M $\operatorname{ImF}$ group ( $\mathrm{n}=7)$; and iv) $3-\mathrm{M} \mathrm{ImF}$ group $(\mathrm{n}=7)$. To investigate effects of ImF on neuroblasts and antioxidant enzymes, animals in each group were sacrificed at the designated times.

Preparation of histological sections. As described previously (16), animals were anesthetized with $60 \mathrm{mg} / \mathrm{kg}$ pentobarbital sodium (JW Pharm. Co., Ltd., Republic of Korea) $(17,18)$ at 1,2 , and 3 months after $I m F$, and perfused transcardially with $0.1 \mathrm{M}$ phosphate buffered saline (PBS, pH 7.4) followed by $4 \%$ paraformaldehyde in $0.1 \mathrm{M}$ phosphate buffer (PB, $\mathrm{pH}$ 7.4). Their brains were removed, and tissues containing hippocampi were cut, cryoprotected and serially sectioned into $25-\mu \mathrm{m}$ frontal sections in a cryostat (Leica, Wetzlar, Germany).

Cresyl violet (CV) histochemistry. CV histochemical staining was performed to investigate cellular distribution and morphology. In brief, according to the method of our previous study (16), One \% of CV acetate (Sigma-Aldrich; Merck KGaA, Darmstadt, Germany) was dissolved in distilled water (DW), and glacial acetic acid was added to this solution. Sections of each group were mounted on gelatin-coated microscopy slides, stained with CV solution and dehydrated with serial ethanol. Finally, the stained sections were covered with Canada balsam (Kanto, Tokyo, Japan).

Immunohistochemistry. In brief, according to our published method (19), sheep anti-SOD1 (1:1,200; Calbiochem, San Diego, CA, USA), sheep anti-SOD2 (1:1,200; Calbiochem), rabbit anti-CAT (1:250; Abcam, Cambridge, MA, USA), rabbit anti-Ki-67 (1:250; Abcam), and rabbit anti-DCX (1:5,500; Abcam), were used as primary antibodies. Sections of each group were sequentially treated with $0.3 \% \mathrm{H}_{2} \mathrm{O}_{2}$ for 40 min and $10 \%$ normal goat serum for $40 \mathrm{~min}$. The treated sections were incubated with each primary antibody overnight at $5^{\circ} \mathrm{C}$. The reacted sections were exposed to biotinylated goat anti-rabbit, rabbit anti-sheep, or goat anti-mouse IgG (1:300; Vector Laboratories, Inc., Burlingame, CA, USA) and streptavidin peroxidase complex (1:300; Vector Laboratories, Inc.). Finally, the reacted sections were visualized by visualizing with 3, 3'-diaminobenzidine tetrahydrochloride (in $0.05 \mathrm{M}$ Tris- $\mathrm{HCl}$ buffer, $\mathrm{pH}$ 7.2).

Data analysis. First, we quantitatively analyzed SOD1, SOD2, and CAT immunoreactivities according to our published method (20). In brief, we selected six sections like the above-mentioned method. Images of SOD1, SOD2, and CAT immunoreactive structures were captured from the dentate gyrus through an AxioM1 light microscope (Carl Zeiss, Göttingen, Germany) equipped with a camera (Axiocam, Carl Zeiss, Germany) connected to a PC monitor. The taken images were calibrated into an array of $512 \times 512$ pixels under $\times 10$ primary magnification. Each immunoreactivity was measured by a $0-255$ gray scale system and evaluated by optical density (OD), which was obtained after transformation of the mean gray level using a formula, $\mathrm{OD}=\log (255 /$ mean gray level). A ratio of the OD was calibrated as \% (relative OD, ROD) using Adobe Photoshop version 8.0 and analyzed using Image J 1.46 software (National Institutes of Health, Bethesda, 
MD, USA). A ratio of the ROD was calibrated as $\%$, with the control group designated as $100 \%$.

Second, we analyzed numbers of Ki67 and DCX positive cells according to our published method (19). In brief, we selected six sections from each gerbil with $140-\mu \mathrm{m}$ interval according to antero-posterior (AP) -1.4 to $-2.2 \mathrm{~mm}$ of the gerbil brain atlas. We took images of the cells from the dentate gyrus through an AxioM1 light microscope (Carl Zeiss,) equipped with a camera (Axiocam; Carl Zeiss) connected to a PC monitor. Cell counts were carried out by averaging the total number of Ki67 and DCX positive cells from all sections taken from each animal by using an image analyzing system (software: Optimus 6.5, CyberMetrics, Scottsdale, AZ, USA).

Statistical analysis. Data are expressed as the means \pm standard error of the mean. Differences of the mean number of immunoreactive structures among the groups was statistically analyzed with one-way analysis of variance followed by post hoc Tukey's test using GraphPad Instat (Instat Statistics; GraphPad Software Inc., La Jolla, CA, USA). P $<0.05$ was considered to indicate a statistically significant difference.

\section{Results}

Body weight. In the control group, body weight was slightly increased for 3 months (Fig. 1). In the ImF groups, body weight was also gradually increased over time. However, body weight between the control and ImF groups was not significantly different (Fig. 1).

\section{Antioxidants immunoreactivities}

SOD1 immunoreactivity. In the control group, SOD1 immunoreactivity was found in cells in the granule cells layer (GCL) and polymorphic layer (PL) of the dentate gyrus (Fig. 2A). In the 1-M ImF group, SOD1 immunoreactivity in the cells was not significantly different compared to that in the control group (Fig. 2B). However, in the 2-M ImF group, SOD1 immunoreactivity in the cells was significantly increased by 56.7 and $43.8 \%$ compared to that in the control and 1-M ImF groups, respectively (Fig. 2C). In the 3-M ImF group, SOD1 immunoreactivity was reduced to the level of the control group (Fig. 2D). SOD1 immunoreactivity in the dentate gyrus in the control and ImF groups was shown in Fig. 2E.

SOD2 immunoreactivity. In the control group, weak SOD2 immunoreactivity was shown in cells in the GCL and PL of the dentate gyrus (Fig. 2F). One month of ImF, SOD2 immunoreactivity was significantly increased by $103.8 \%$ compared to that in the control group (Fig. 2G). Two and three months of $\operatorname{ImF}$, SOD2 immunoreactivity in the cells was decreased by 60.9 and $95.1 \%$, respectively, compared to the $1-\mathrm{M} \mathrm{ImF}$ group, showing that SOD2 immunoreactivity after three months of ImF 3 was similar to that in the control group (Fig. $2 \mathrm{H}$ and I). SOD2 immunoreactivity in the dentate gyrus in the control and ImF groups was shown in Fig. 2J.

CAT immunoreactivity. In the control group, very weak CAT immunoreactivity was observed in cells in the GCL and PL of the dentate gyrus (Fig. 2K). In the 1-M ImF and 2-M ImF groups, CAT immunoreactivity in the cells was significantly

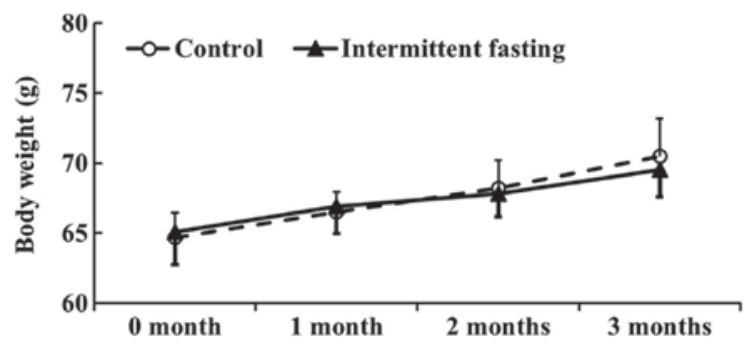

Figure 1. Body weight. Body weight changes in the control and ImF groups over 1, 2 and 3 months. In the control and ImF groups, body weight slightly increased over time, although there was no significant difference between the two groups ( $n=7$ at each point in time). Bars indicate the mean \pm standard error of the mean. ImF, intermittent fasting.

increased by 183.9 and $123.0 \%$, respectively, compared to that in the control group (Fig. 2L and M). In the 3-M ImF group, CAT immunoreactivity in the cells was markedly decreased by $108.3 \%$ compared to that in the $2-\mathrm{M} \mathrm{ImF}$ group, showing that there was no significant difference from the control group (Fig. 2N). CAT immunoreactivity in the dentate gyrus in the control and ImF groups was shown in Fig. $2 \mathrm{O}$.

Cell proliferation. In the control group, a few Ki67 positive cells were mainly located in the subgranular zone $(\mathrm{SgZ})$ of the dentate gyrus (Fig. 3A). In the 1-M ImF group, many Ki67 positive cells were observed in the $\mathrm{SgZ}$, and the number of Ki67 positive cells was significantly increased by $250.0 \%$ compared to that in the control group (Fig. 3B). In the 2-M ImF group, Ki67 positive cells were scattered in the $\mathrm{SgZ}$, showing that the Ki67 positive cells were significantly increased in number by $128.6 \%$ compared to those in the control group (Fig. 3C). At 3 months of ImF, the number of Ki67 positive cells was significantly increased by $185.7 \%$ compared to that in the control group (Fig. 3D). However, Ki67 positive cells in the 2-M ImF and 3-M ImF groups were significantly decreased in number compared to that in the 1-M ImF group (Fig. 3E).

Neuroblast differentiation. In the control group, DCX positive cells were mainly observed in the $\mathrm{SgZ}$, and their processes projected to the GCL (Fig. 4A). In the 1-M ImF group, DCX positive cells were also found in the $\mathrm{SgZ}$, and their number was significantly increased by $41.1 \%$ compared to that in the control group (Fig. 4B). In the 2-M ImF group, DCX positive cells were also significantly increased in number by $32.4 \%$ compared to that in the control group (Fig. 4C). At 3 months of ImF, the number of DCX positive cells was slightly decreased compared to that in the 2-M ImF group (Fig. 4D). The number of DCX positive cells in the control and ImF groups was shown in Fig. 4E.

In this study, according to our previous studies $(17,21)$, we categorized DCX positive cells into types $\mathrm{a}, \mathrm{b}$ and $\mathrm{c}$ based on the morphology and complexity of the dendrites: Dendrites of type a cells were absent or shorter than soma size, type b cells had one primary dendrite with one branch, and type c had dendrites with highly arborized branches that extended into the upper two-thirds of the molecular layer (Fig. 5A). In the control group, the proportion of dendritic types of DCX positive cells was relatively uniform (Fig. 5B and C). However, in the 1-M ImF and 2-M ImF groups, the ratio of the type $\mathrm{c}$ dendrites was increased slightly (Fig. 5B, D and E). In the 

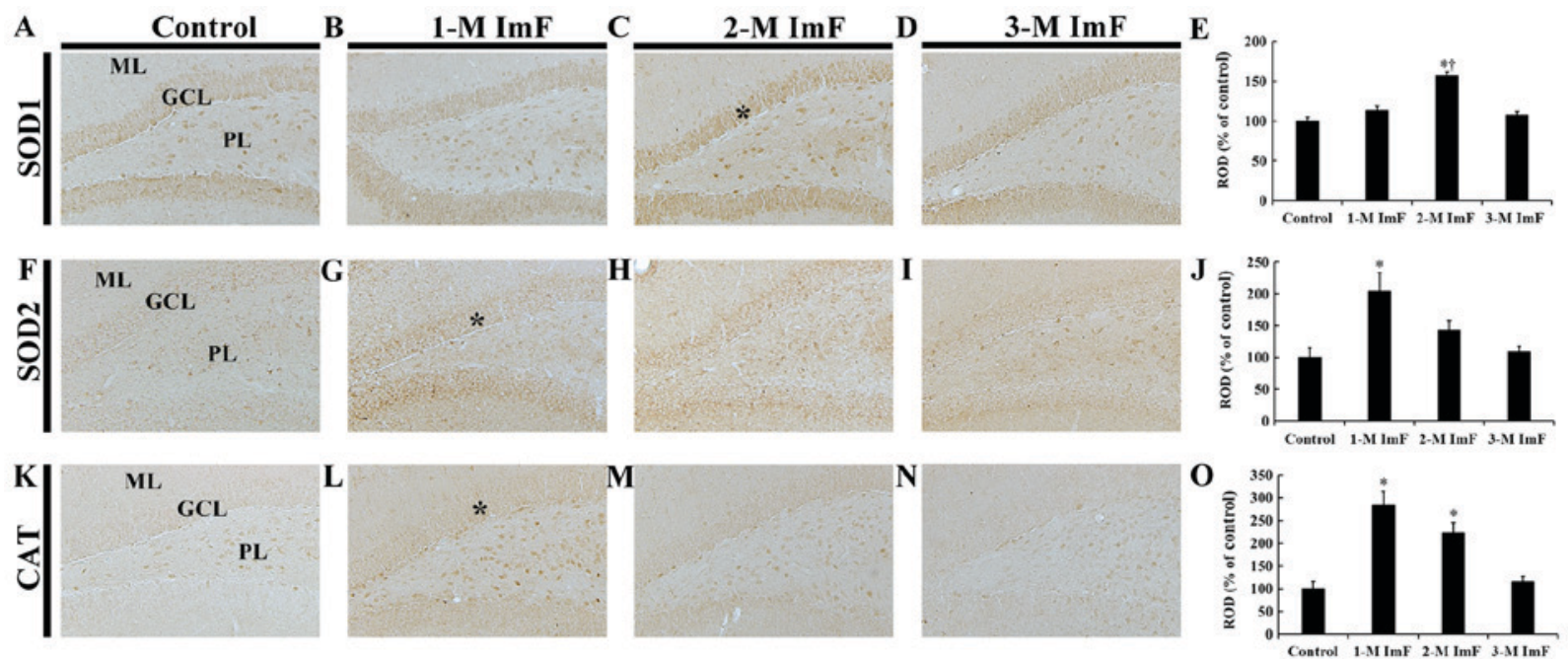

Figure 2. SOD1, SOD2 and CAT immunohistochemistry. Immunoreactivities for (A-E) SOD1, (F-J) SOD2 and (K-O) CAT of the control and ImF groups. In the control group, the SOD1, SOD2 and CAT immunoreactivities were observed in cells in the GCL and PL. SOD1 immunoreactivity was significantly increased in the GCL (asterisk in C) in the 2-M ImF group. SOD2 immunoreactivity was significantly increased in the GCL (asterisk in G) at 1 month of ImF. CAT immunoreactivity was significantly increased in the GCL (asterisk in L) in the 1-M ImF group. Magnification, x10; Scale bar=100 $\mu \mathrm{m}$. (E, J and O) ROD of (E) SOD1, (J) SOD2 and (O) CAT immunoreactive structures. Bars indicate the mean \pm standard error of the mean ( $\mathrm{n}=7$ per group). ${ }^{*} \mathrm{P}<0.05$ vs. the control group; ${ }^{\dagger} \mathrm{P}<0.05$ vs. the $1-\mathrm{M}$ ImF group. GCL, granule cell layer; $\mathrm{ML}$, molecular layer; ROD, relative optical density; SOD, superoxide dismutase; CAT, catalase; PL, polymorphic layer; ImF, intermittent fasting.

A

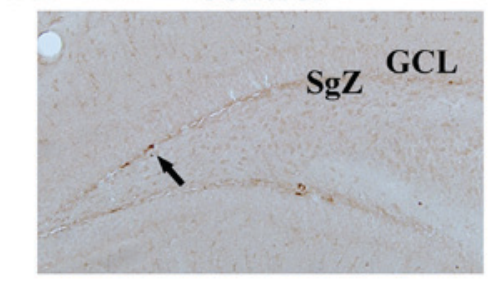

C

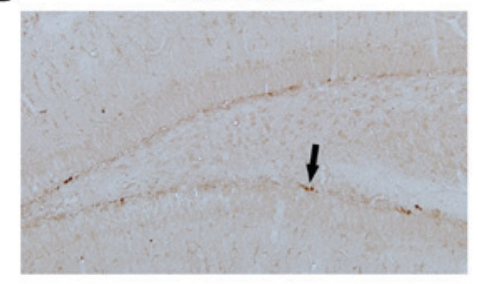

$\mathbf{E}$

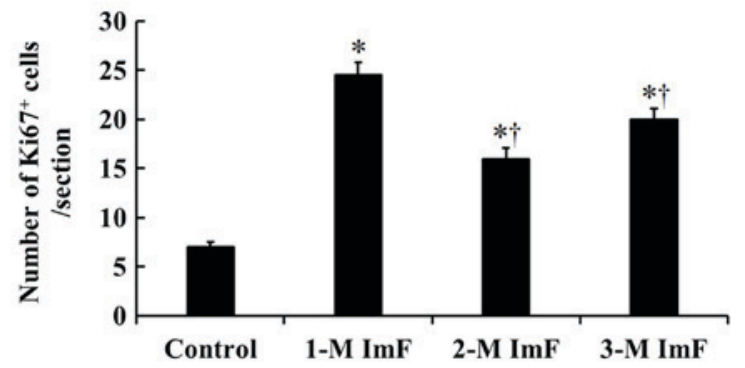

Figure 3. Ki67 immunohistochemistry. Ki67 immunoreactive cells in the dentate gyrus of the (A) control, and the (B) 1-M, (C) 2-M and (D) 3-M ImF groups. Ki67 positive cells (arrows) were mainly distributed in the SgZ. At 1 to 3 months of ImF, the number of Ki67 positive cells was significantly increased when compared with the control group. Magnification, x10; Scale bars $=100 \mu \mathrm{m}$. (E) Number of Ki67 positive cells per section. Bars indicate the mean \pm standard error of the mean ( $\mathrm{n}=7$ per group). ${ }^{\mathrm{P}} \mathrm{P}<0.05$ vs. the control group; ${ }^{\dagger} \mathrm{P}<0.05$ vs. the $1-\mathrm{M} \mathrm{ImF}$ group. SgZ, subgranular zone; ImF, intermittent fasting.

3-M ImF group, the ratio of the type $b$ dendrites was significantly decreased, and the ratio of the type c dendrites was significantly increased compared to that in the control group (Fig. 5B and F).
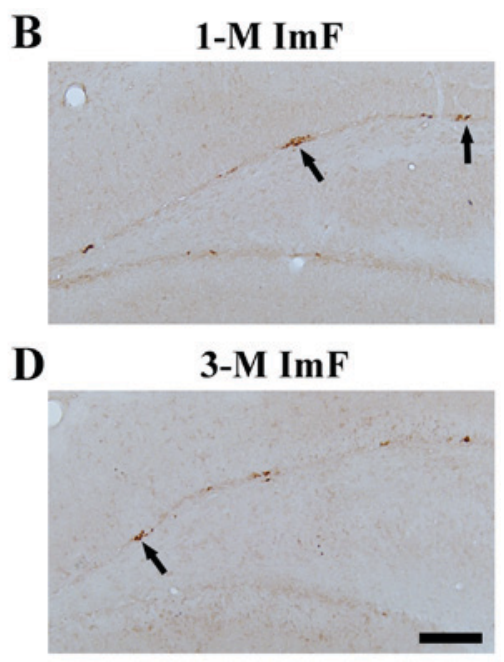

\section{Discussion}

In the present study, we investigated effects of ImF on endogenous antioxidant enzymes such as SOD1, SOD2 and CAT as 
A

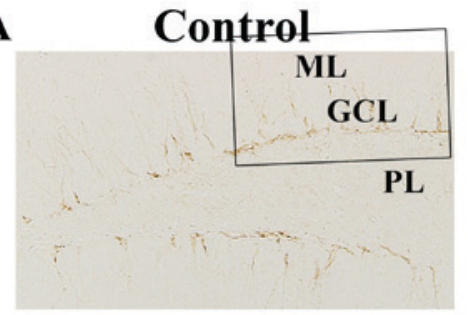

C

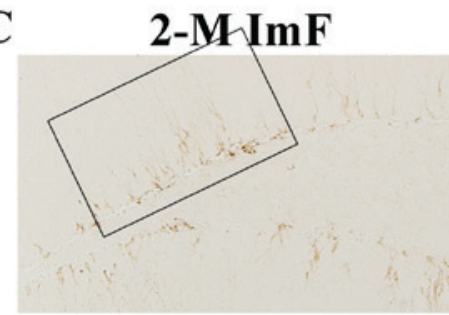

B

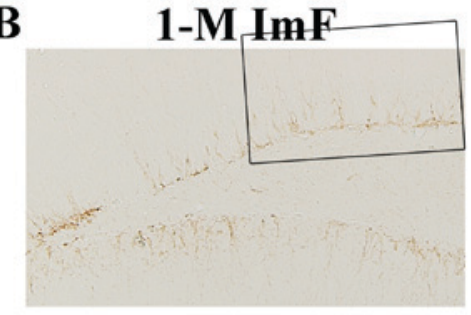

D

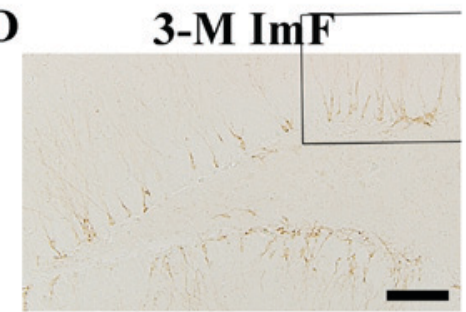

E

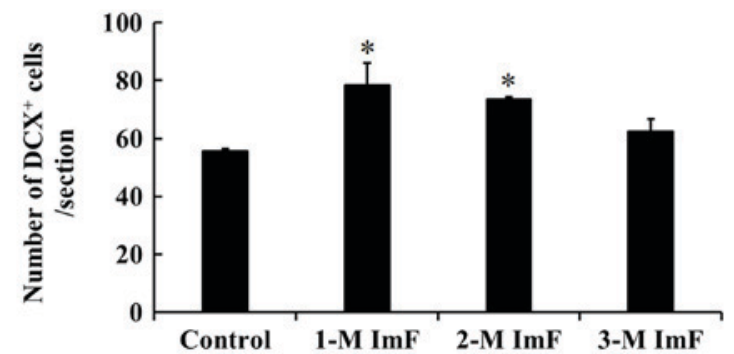

Figure 4. DCX immunohistochemistry. DCX immunoreactive cells in the dentate gyrus of the (A) control, and the (B) 1-M, (C) 2-M and (D) 3-M ImF groups. DCX positive cells were easily detected in the GCL. At 1 and 2 months of ImF, the number of DCX positive cells was significantly increased. Magnification, $\mathrm{x} 10$; Scale bars $=100 \mu \mathrm{m}$. (E) Number of DCX positive cells per section. Bars indicate the mean \pm standard error of the mean ( $\mathrm{n}=7$ per group). ${ }^{*} \mathrm{P}<0.05 \mathrm{vs}$. the control group. GCL, granule cell layer; ML, molecular layer; DCX, doublecortin; ImF, intermittent fasting.

A

Types of $\mathrm{DCX}^{+}$cells

○

(a)

C

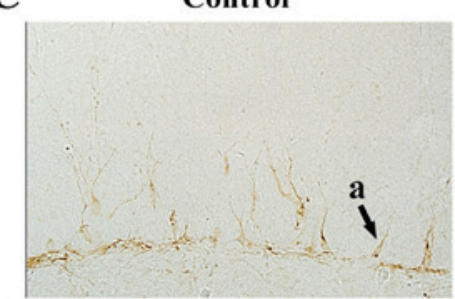

$\mathbf{E}$

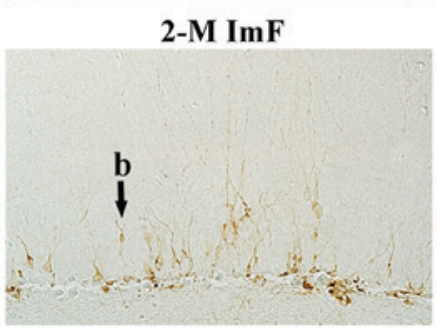

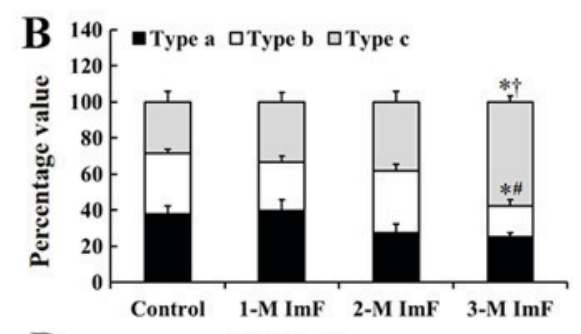

D

1-M ImF

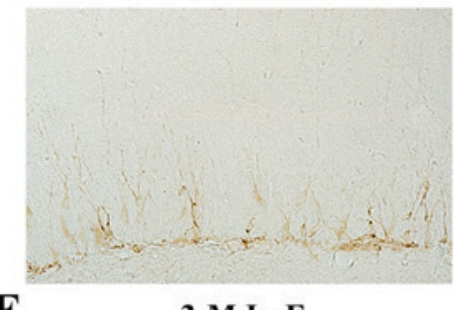

$\mathbf{F}$

3-M ImF

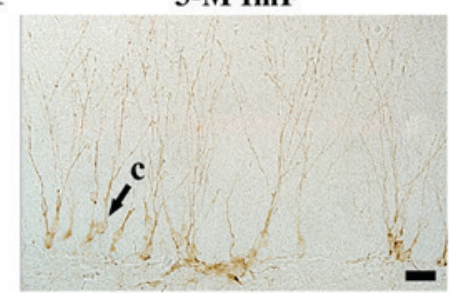

Figure 5. Types of DCX immunoreactive cells and higher magnification of DCX immunohistochemistry. (A) Types of dendrites of DCX positive cells [(a) cells without dendrites or shorter dendrites than the soma size; (b) cells with one primary dendrite with one branch; and (c) cells with much more branches reaching the molecular layer]. (B) Percentage value in types a-c of DCX positive cells per section. Bars indicate the mean \pm standard error of the mean ( $\mathrm{n}=7 \mathrm{per}$ group). ${ }^{*} \mathrm{P}<0.05$ vs. the control group; ${ }^{\mathrm{P}} \mathrm{P}<0.05$ vs. the $1-\mathrm{M}$ ImF group; ${ }^{\mathrm{P}} \mathrm{P}<0.05$ vs. the $2-\mathrm{M}$ ImF group. Higher magnification of the rectangular areas presented in Fig. 3A-D in the (C) control and the (D) 1-M, (E) 2-M and (F) 3-M ImF groups. At 3 months of ImF, the ratio of type c dendrites of DCX positive cells was significantly increased when compared with that observed in the control group, and dendrites (arrows) were highly branched and long, which projected into the granule cell layer. Magnification, x10; Scale bars=100 $\mu \mathrm{m}$. DCX, doublecortin; ImF, intermittent fasting. 
well as cell proliferation, neuroblast differentiation and their dendrite arborization according to the period of $\mathrm{ImF}$ in the gerbil dentate gyrus using immunohistochemistry.

Our results showed that body weight was gradually increased over a period of 3 months in the control and ImF groups in adult gerbils (6-month old), but there was no significant difference between the control and ImF groups. This finding is supported by a previous study which revealed that increased body weight of control and 5-M ImF groups were not significantly different in adult C57BL/6 mice (9-weeks old) (22). On the other hand, body weight in Wistar rats of control and 1-M ImF groups was changed according to age; however, no significant difference was shown between the groups (23). Namely, body weight was progressively increased in young rats (4-month old), whereas slightly decreased in aged rats (24-month old) (23). Furthermore, Li et al (1) reported that, for 11 months of ImF, significant body weight change in adult CD-1 mice (7-week old) appeared after 37 weeks (around 9 months) of ImF. Based on these previous studies and our results, it is postulated that weight change after alternate day ImF regimen might be affected by age and fasting period.

In this study, 1 and/or 2 months of ImF regimen significantly increased SOD1, SOD2 and CAT immunoreactivities in the granule cell and polymorphic layer of the gerbil dentate gyrus. In Walsh's et al (24) review article, they reported that the production of mitochondrial reactive oxidative stress was reduced in the brain as well as in the heart, kidneys, liver, and skeletal muscle: In particular, SOD and CAT activities in the brain were increased after ImF in $20-30 \%$ of studies.

Newly generated neurons in the $\mathrm{SgZ}$ of the dentate gyrus go through multi-stages of morphological development: The growth of dendrites and axons of immature neurons occurs about 1 week after cell birth, and basic structural and physiological characteristics of adult-born neurons become similar to those of mature neurons at around 2 months in the dentate gyrus (8). In the present study, we observed that ImF significantly increased numbers of Ki67 positive (newly generated) cells for 3 months of ImF as well as numbers of DCX positive cells (neuroblasts) for 2 months of $\mathrm{ImF}$ in the $\mathrm{SgZ}$ of the dentate gyrus. In addition, at 3 months of ImF, the proportion of DCX positive neuroblasts with tertiary dendrites was significantly increased. Previous investigators have reported similar results that show that the number of newly generated progenitor cells $\left(\mathrm{BrdU}^{+}\right)$, which differentiate to mature neurons $\left(\mathrm{BrdU}^{+} / \mathrm{MAP}^{+}\right)$, in the dentate gyrus are significantly increased after 3 months of $\operatorname{ImF}(9,10)$. Furthermore, fasting mimicking diet (alternating fasting designed as low-calorie consumption only for 4 days of diet) also increases numbers of new born progenitor cells $\left(\mathrm{BrdU}^{+}\right)$and newly generated immature neurons $\left(\mathrm{BrdU}^{+} / \mathrm{DCX}^{+}\right)$in aged $\mathrm{C} 57 \mathrm{Bl} / 6$ mice (23-month old) as well as increased DCX positive neuroblasts in number and dendrites maturation in adult CD-1 mice (6-month old) (25). Taken together with our present findings and the previous studies, continued $\mathrm{ImF}$ regimen is likely to increase cell proliferation, neuroblast differentiation and complexity of neuroblast dendrites in the gerbil hippocampal dentate gyrus.

Previous studies have revealed that antioxidant enzymes are closely linked to increased neurogenesis. Fishman et al (26) have demonstrated that SOD1 and SOD2 knockout mice show a significant reduction in numbers of newly born neurons in the $\mathrm{SgZ}$ of the dentate gyrus. In addition, mitochondrial CAT overexpressed transgenic mice show an increase of basal neurogenesis in the dentate gyrus (27) as well as a significant improvement in dendritic arborization of granular cells against proton irradiation (28). Furthermore, transgenic mice with mitochondrial CAT overexpression show a tendency to increase dendritic complexity and synaptic integrity of subiculum neurons (29). Furthermore, Dias et al (30) have extensively reviewed previous researches and summarized that dietary polyphenols, which known to be the most abundant antioxidant in foods, exert the improvement of NPCs proliferation and neuronal differentiation. Taken together, our present results, which are described in the 3rd paragraph, suggest that ImF could affect neuronal differentiation and maturation by increasing SOD1, SOD2, and CAT expressions in the gerbil dentate gyrus.

To sum up, findings in this study indicate that $\operatorname{ImF}$ regimen enhances cell proliferation, neuroblast differentiation and dendrites maturation by increasing SOD1, SOD2, and CAT immunoreactivities in the gerbil hippocampal dentate gyrus.

\section{Acknowledgements}

\section{Not applicable.}

\section{Funding}

The present study was supported by Basic Science Research Program through the National Research Foundation of Korea (NRF) funded by the Ministry of Education (grant no. NRF-2015R1D1A1A01059728), and by Basic Science Research Program through the National Research Foundation of Korea (NRF) funded by the Ministry of Science, ICT \&Future Planning (NRF-2017R1A2B4009079).

\section{Availability of data and materials}

All data generated or analyzed during this study are included in this published article.

\section{Authors' contribution}

BS, MS, HK, TL, CP and YP performed the experiments. JP, JL, JY, CL and IH analyzed and interpreted the data. JA, MW and YL made substantial contributions to the conception and design of the study, and were involved in drafting and revising the manuscript, and interpreting the data. All Authors read and approved the final manuscript.

\section{Ethics approval and consent to participate}

The protocol of this experiment was approved by the Institutional Animal Care and Use Committee (IACUC) at Kangwon National University (approval number, KW-180124-1; Gangwon, Republic of Korea).

\section{Patient consent for publication}

Not applicable. 


\section{Competing interests}

The authors declare that they have no competing interests.

\section{References}

1. Li L, Wang Z and Zuo Z: Chronic intermittent fasting improves cognitive functions and brain structures in mice. PLoS One 8 e66069, 2013.

2. Jeong JH, Yu KS, Bak DH, Lee JH, Lee NS, Jeong YG, Kim DK, Kim JJ and Han SY: Intermittent fasting is neuroprotective in focal cerebral ischemia by minimizing autophagic flux disturbance and inhibiting apoptosis. Exp Ther Med 12: 3021-3028, 2016.

3. Arumugam TV, Phillips TM, Cheng A, Morrell CH, Mattson MP and Wan R: Age and energy intake interact to modify cell stress pathways and stroke outcome. Ann Neurol 67: 41-52, 2010.

4. Mladenovic Djordjevic A, Perovic M, Tesic V, Tanic N, Rakic L, Ruzdijic S and Kanazir S: Long-term dietary restriction modulates the level of presynaptic proteins in the cortex and hippocampus of the aging rat. Neurochem Int 56: 250-255, 2010

5. Ma L, Wang R, Dong W and Zhao Z: Caloric restriction can improve learning and memory in $\mathrm{C} 57 / \mathrm{BL}$ mice probably via regulation of the AMPK signaling pathway. Exp Gerontol 102: 28-35, 2018.

6. Gage FH: Mammalian neural stem cells. Science 287: 1433-1438, 2000.

7. Lee J, Duan W and Mattson MP: Evidence that brain-derived neurotrophic factor is required for basal neurogenesis and mediates, in part, the enhancement of neurogenesis by dietary restriction in the hippocampus of adult mice. J Neurochem 82: 1367-1375, 2002.

8. Deng W, Aimone JB and Gage FH: New neurons and new memories: How does adult hippocampal neurogenesis affect learning and memory? Nat Rev Neurosci 11: 339-350, 2010.

9. Lee J, Duan W, Long JM, Ingram DK and Mattson MP: Dietary restriction increases the number of newly generated neural cells and induces BDNF expression, in the dentate gyrus of rats. J Mol Neurosci 15: 99-108, 2000.

10. Lee J, Seroogy KB and Mattson MP: Dietary restriction enhances neurotrophin expression and neurogenesis in the hippocampus of adult mice. J Neurochem 80: 539-547, 2002.

11. Zelko IN, Mariani TJ and Folz RJ: Superoxide dismutase multigene family: A comparison of the CuZn-SOD (SOD1), Mn-SOD (SOD2), and EC-SOD (SOD3) gene structures, evolution and expression. Free Radic Biol Med 33: 337-349, 2002.

12. Chelikani P, Fita I and Loewen PC: Diversity of structures and properties among catalases. Cell Mol Life Sci 61: 192-208, 2004

13. Faiz M, Acarin L, Peluffo H, Villapol S, Castellano B and Gonzalez B: Antioxidant Cu/Zn SOD: Expression in postnatal brain progenitor cells. Neurosci Lett 401: 71-76, 2006

14. Ostadsharif M, Ghaedi K, Hossein Nasr-Esfahani M, Mojbafan M, Tanhaie S, Karbalaie K and Baharvand $\mathrm{H}$ : The expression of peroxisomal protein transcripts increased by retinoic acid during neural differentiation. Differentiation 81: 127-132, 2011.

15. Tajes M, Gutierrez-Cuesta J, Folch J, Ortuño-Sahagun D, Verdaguer E, Jiménez A, Junyent F, Lau A, Camins A and Pallàs M Neuroprotective role of intermittent fasting in senescence-accelerated mice P8 (SAMP8). Exp Gerontol 45: 702-710, 2010.

16. Ahn JH, Shin BN, Park JH, Kim IH, Cho JH, Chen B, Lee TK, Tae HJ, Lee JC, Cho JH, et al: Long-term observation of neuronal degeneration and microgliosis in the gerbil dentate gyrus after transient cerebral ischemia. J Neurol Sci 363: 21-26, 2016.

17. Yan BC, Park JH, Chen BH, Cho JH, Kim IH, Ahn JH, Lee JC, Hwang IK, Cho JH, Lee YL, et al: Long-term administration of scopolamine interferes with nerve cell proliferation, differentiation and migration in adult mouse hippocampal dentate gyrus, but it does not induce cell death. Neural Regen Res 9: 1731-1739, 2014.
18. Carpenter JW: Exotic animal formulary (ED 4). J Exotic Pet Med 22: 308-309, 2013.

19. Chen BH, Ahn JH, Park JH, Song M, Kim H, Lee TK, Lee JC, Kim YM, Hwang IK, Kim DW, et al: Rufinamide, an antiepileptic drug, improves cognition and increases neurogenesis in the aged gerbil hippocampal dentate gyrus via increasing expressions of IGF-1, IGF-1R and p-CREB. Chem Biol Interact 286: 71-77, 2018.

20. Lee TK, Park JH, Ahn JH, Shin MC, Cho JH, Bae EJ, Kim YM, Won $\mathrm{MH}$ and Lee $\mathrm{CH}$ : Pretreated duloxetine protects hippocampal CA1 pyramidal neurons from ischemia-reperfusion injury through decreases of glial activation and oxidative stress. J Neurol Sci 370: 229-236, 2016.

21. Chen BH, Yan BC, Park JH, Ahn JH, Lee DH, Kim IH, Cho JH, Lee JC, Kim SK, Lee B, et al: Aripiprazole, an atypical antipsychotic drug, improves maturation and complexity of neuroblast dendrites in the mouse dentate gyrus via increasing superoxide dismutases. Neurochem Res 38: 1980-1988, 2013.

22. Anson RM, Guo Z, de Cabo R, Iyun T, Rios M, Hagepanos A, Ingram DK, Lane MA and Mattson MP: Intermittent fasting dissociates beneficial effects of dietary restriction on glucose metabolism and neuronal resistance to injury from calorie intake. Proc Natl Acad Sci USA 100: 6216-6220, 2003.

23. Vasconcelos AR, Kinoshita PF, Yshii LM, Marques Orellana AM, Böhmer AE, de SáLima L, Alves R, AndreottiDZ, Marcourakis T, Scavone $\mathrm{C}$ and Kawamoto EM: Effects of intermittent fasting on age-related changes on $\mathrm{Na}, \mathrm{K}$-ATPase activity and oxidative status induced by lipopolysaccharide in rat hippocampus. Neurobiol Aging 36: 1914-1923, 2015.

24. Walsh ME, Shi Y and Van Remmen H: The effects of dietary restriction on oxidative stress in rodents. Free Radic Biol Med 66: 88-99, 2014.

25. Brandhorst S, Choi IY, Wei M, Cheng CW, Sedrakyan S, Navarrete G, Dubeau L, Yap LP, Park R, Vinciguerra M, et al: A periodic diet that mimics fasting promotes multi-system regeneration, enhanced cognitive performance, and healthspan. Cell Metab 22: 86-99, 2015.

26. Fishman K, Baure J, Zou Y, Huang TT, Andres-Mach M, Rola R, Suarez T, Acharya M, Limoli CL, Lamborn KR and Fike JR: Radiation-induced reductions in neurogenesis are ameliorated in mice deficient in CuZnSOD or MnSOD. Free Radic Biol Med 47: 1459-1467, 2009.

27. Liao AC, Craver BM, Tseng BP, Tran KK, Parihar VK, Acharya MM and Limoli CL: Mitochondrial-targeted human catalase affords neuroprotection from proton irradiation. Radiat Res 180: 1-6, 2013.

28. Parihar VK, Allen BD, Tran KK, Chmielewski NN, Craver BM, Martirosian V, Morganti JM, Rosi S, Vlkolinsky R, Acharya MM, et al: Targeted overexpression of mitochondrial catalase prevents radiation-induced cognitive dysfunction. Antioxid Redox Signal 22: 78-91, 2015.

29. Chmielewski NN, Caressi C, Giedzinski E, Parihar VK and Limoli CL: Contrasting the effects of proton irradiation on dendritic complexity of subiculum neurons in wild type and MCAT mice. Environ Mol Mutagen 57: 364-371, 2016.

30. Dias GP, Cavegn N, Nix A, do Nascimento Bevilaqua MC, Stangl D, Zainuddin MS, Nardi AE, Gardino PF and Thuret S: The role of dietary polyphenols on adult hippocampal neurogenesis: Molecular mechanisms and behavioural effects on depression and anxiety. Oxid Med Cell Longev 2012: 541971, 2012.

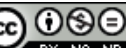

This work is licensed under a Creative Commons Attribution-NonCommercial-NoDerivatives 4.0 International (CC BY-NC-ND 4.0) License. 